\title{
Are Eurozone Fixed Income Markets Integrated? An Analysis Based on Wavelet Multiple Correlation and Cross Correlation
}

\author{
Arif Billah Dar ${ }^{1}$ and Firdous Ahmad Shah ${ }^{2}$ \\ ${ }^{1}$ Department of Economic Environment and Strategy, IMT, Ghaziabad 201001, India \\ ${ }^{2}$ Department of Mathematics, University of Kashmir, South Campus, Anantnag, Jammu and Kashmir 192101, India
}

Correspondence should be addressed to Firdous Ahmad Shah; fashahku@gmail.com

Received 2 June 2014; Accepted 22 July 2014; Published 28 August 2014

Academic Editor: João Ricardo Faria

Copyright ( 2014 A. B. Dar and F. A. Shah. This is an open access article distributed under the Creative Commons Attribution License, which permits unrestricted use, distribution, and reproduction in any medium, provided the original work is properly cited.

\begin{abstract}
This paper investigates the synchronization of fixed income markets within Eurozone countries using the new wavelet based methodology. Conventional wavelet methods that use multivariate set of variables to calculate pairwise correlation and cross correlation lead to spurious correlation due to possible relationships with other variables, amplification of type-1 errors, and results, in the form of large set of erroneous graphs. Given these disadvantages of conventional wavelet based pairwise correlation and crosscorrelation method, we avoid these limitations by using wavelet multiple correlation and multiple cross correlations to analyze the relationships in Eurozone fixed income markets. Our results based on this methodology indicate that Eurozone fixed income markets are highly integrated and this integration grows with timescales, and hence there is almost no scope for independent monetary policy and bond diversification in these countries.
\end{abstract}

\section{Introduction}

On January 1, 1999, a monetary union Known as European Monetary Union (EMU) was formed by group of eleven European Union countries. This was followed by the introduction of euro as the common currency. The European Central Bank began operating and carrying out the common monetary policy, and all EU-11 government bills and bonds began to be denominated in Euro. The introduction of the euro has been one of the most important events for global financial markets in the last decade and it has become one of the three major currencies in the world after its introduction, taking its place alongside the US dollar and the Japanese yen. An immediate consequence of the adoption of the common currency has been the convergence of Eurozone interest rates and the integration of fixed income markets (Hartmann et al. [1]).

A voluminous body of literature has focused on the integration of equity markets (Bartram and Wang [2], Hardouvelis et al. [3], and Diamandis [4]); however, there is relatively scant literature which investigates integration between the bond markets. Synchronization of bond markets may be induced by diverse types of reasons like similarity in monetary policy reactions functions, increased monetary policy coordination, and globalization induced closer alignment of economic fundamentals (Hunter and Simson [5]). Under such synchronized and integrated system of international markets, conduct of monetary policy by central banks becomes even much more challenging. It is generally argued that the financial market globalization is responsible for reducing the scope for independent monetary policy action by strengthening the relationship between national fixed income markets. However, if the long-term interest rates are determined by national rather than international factors, monetary policy makers are still independent in framing the domestic monetary policy (Barassi et al. [6]). This is true not only for large open rather small open economies also. In essence, long-term interest rates are synonymous to other assets prices or policy instruments. They may also be expected to move together when financial markets are substantially deregulated. It is therefore important to determine the extent to which interest rates are synchronized in different countries for a number of reasons. Fujihara and Mougoué [7] underlined several reasons as to why researchers should 
know the integration in financial markets in general and fixed income markets in particular. These include the following. (i) Knowledge of linkages in international financial markets could be useful in knowing how economic disturbances are transmitted between countries. (ii) The integration of international financial markets could affect the conduct of monetary policy. This is because of the fact that the degree of independence of domestic stabilization policies from foreign policies is inversely related to the extent to which domestic real interest rates are linked with foreign rates.

There is a scant but significant literature available on the comovement between long term interest rates and much of this research mainly focuses on cointegration and error correction between the long-term interest rates (see, e.g., Barassi et al. [6], Degennaro et al. [8], Kiran [9], Smith [10], and Yang [11]). However, these econometric methodologies deal with just two time frames: the short and the long run with restrictive assumption of variables being integrated of order one $I$ (1) (nonstationary).

An alternative methodology of correlation is based on wavelets that considers nonstationarity as an inherent tendency of data and has ability to analyze the time series over different time scales. The technique of wavelets has frequently been applied in number of recent works (Dar et al. [12, 13] and Tiwari et al. [14]). Some of the recent work has also focused on the comovement of equity prices in different countries. Rua and Nunes [15] and Raghavan et al. [16], for example, use conventional wavelets to calculate pairwise multiscale correlations of stock returns for different countries. Conventional wavelet based correlation and cross correlation, however, use simple pairwise correlations to analyze the relationship between different time series. This comes with large number of disadvantages; for example, one needs to calculate, plot, and compare a large number of wavelet correlation and cross-correlation graphs (In this study we are using wavelets to decompose the eleven Eurozone stock returns into four detail components. Using the pairwise wavelet correlation and cross correlation, we would have end up with 55 wavelet correlation graphs and 220 cross-correlation graphs.). Moreover, within the multivariate context, pairwise correlation coefficient could be spurious due to possible relationships with the stock prices of other countries. Finally, pairwise correlations in multiscale are prone to amplify the type-1 errors due to experimentwise error rate (see Cohen et al. [17]). For instance, at a given wavelet scale, $5 \%$ level of significance doing pairwise wavelet correlation significance tests with nine unrelated series of stock returns (number of stock returns we are analyzing in our study) will inflate the chance of type-1 error to 1 - (1 $\alpha)^{55}=0.94$. This increases the chance of finding significant correlation to $94 \%$ at given wavelet scale somewhere among 55 tests instead of mere $5 \%$.

Given these disadvantages of conventional wavelet correlation and cross correlation, this study uses the wavelet multiple correlation and cross correlation recently proposed by Fernández-Macho [18] to analyze the integration in the Eurozone bond market over different timescales. The proposed methodology is useful at least in three respects.
Overall correlation within the multivariate set of different timescales in stock markets can be viewed just in two plots, spurious correlation obtained from the pairwise correlations within the multivariate set of stock returns could be avoided, and finally, the proposed methodology could be useful in providing the protection against type- 1 errors. Our results based on this methodology indicate that the Eurozone bond markets are highly integrated at studied timescales and this integration grows stronger with lower frequencies (higher time scales).

The remainder of the paper is organized as follows. The motivation and methodology used in the paper is described in Section 2. Section 3 gives the data description and discussion of results and finally, Section 4 draws the conclusions and policy implications.

\section{Motivation and Methodology}

Much of the empirical research on integration in crosscountry bond markets focuses on the short run and long run relationship between long term interest rates within cointegration and error correction frame work. One of the limitations of these models is that the definition of long run and short run is narrow. Moreover, these models have been designed to deal with just two time frames: the short and the long run with restrictive assumption of variables being integrated of order one $I(1)$. However, central banks do not operate at two timescales only; rather, they operate at more than two timescales separately (see Aguiar-Conraria et al. [19]). This operation of central banks at different timescales also leads to heterogeneous behaviour of long term interest rates across different timescales. Therefore, it becomes imperative to analyze the integration of long term interest rates at different timescales (frequencies).

2.1. The Wavelet Transforms. To overcome the problems pointed above, wavelet analysis has been proposed. Wavelet analysis shares several features in common with Fourier analysis but has the advantage of capturing features in the underlying series that vary across both time and frequency. Wavelets are mathematical functions that decompose data into different frequency components, after which each component is studied with a resolution matched to its scale. These functions are generated by dilations and translations of a single function called a mother wavelet. More explicitly, wavelets are defined as

$$
\psi_{a, b}(t)=\frac{1}{\sqrt{a}} \psi\left(\frac{t-b}{a}\right), \quad a \neq 0, b \in \mathbb{R}
$$

where $1 / \sqrt{a}$ is a normalization factor to ensure that wavelets are comparable across scales and time series. Here, the dilation factor " $a$ " controls the width of the wavelet: it stretches it into a long wavelet function to measure the low frequency movements (long-run cycles), and it is compressed into a short wavelet function to measure the high frequency movements whereas the translation factor " $b$ " determines the time location of the wavelet. To be a mother wavelet, 
$\psi(t)$ must obey a basic rule, known as the wavelet admissibility condition:

$$
0<C_{\psi}=\int_{0}^{\infty} \frac{|\widehat{\psi}(\xi)|^{2}}{|\xi|} \mathrm{d} \xi<\infty
$$

where $\widehat{\psi}(\xi)$ is the Fourier transform of $\psi(t)$. To guarantee that $C_{\psi}<\infty$, the wavelet function must satisfy the conditions $\int_{-\infty}^{\infty} \psi(t) \mathrm{d} t=0$ and $\int_{-\infty}^{\infty}|\psi(t)|^{2} \mathrm{~d} t=1$. The first condition means that $\psi(t)$ must be an oscillatory function with zero mean and the second condition ensures that the wavelet function has unit energy.

The Continuous wavelet transform (CWT) of a time series $x(t)$ with respect to $\psi(t)$ is given by the following convolution:

$$
W_{x}(a, b)(t)=\frac{1}{\sqrt{a}} \int_{-\infty}^{\infty} x(t) \overline{\psi\left(\frac{t-b}{a}\right)} \mathrm{d} t .
$$

The inverse wavelet transform can be defined so that $x(t)$ can be reconstructed by means of the formula

$$
x(t)=\frac{1}{C_{\psi}} \int_{-\infty}^{\infty} \int_{-\infty}^{\infty} \frac{1}{\sqrt{a}} \psi\left(\frac{t-b}{a}\right) W_{x}(a, b)(t) \frac{\mathrm{d} a \mathrm{~d} b}{a^{2}} .
$$

Provided $C_{\psi}$ satisfies the admissibility condition (2), the discrete wavelet transform (DWT) (Daubechies [20]), in particular, allows the decomposition of a signal into components of different frequency. There are two basic wavelet functions, the father wavelet $\phi$ and the mother wavelet $\psi$. The formal definitions of the father and mother wavelets are the functions

$$
\begin{array}{ll}
\phi_{j, k}(t)=2^{j / 2} \phi\left(2^{j} t-k\right), & j, k \in \mathbb{Z}, \\
\psi_{j, k}(t)=2^{j / 2} \psi\left(2^{j} t-k\right), & j, k \in \mathbb{Z},
\end{array}
$$

where $j=1, \ldots, J$ in a $J$ level decomposition and $J$ denotes the maximum scale sustainable by the number of data points and the two types of wavelets stated above satisfiy

$$
\int_{-\infty}^{\infty} \phi(t) \mathrm{d} t=1, \quad \int_{-\infty}^{\infty} \psi(t) \mathrm{d} t=0
$$

Thus, the scaling function integrates to 1 and reconstructs the smooth and low-frequency parts of a signal, whereas the wavelet function integrates to 0 and describes the detailed and high-frequency parts of a signal. In this way, by applying a $J$ level multiresolution decomposition analysis we can provide a complete reconstruction of the signal partitioned into a set of $J$-frequency components so that each component corresponds to a particular range of frequencies.

The wavelet representation of a discrete signal $x(t)$ in $L^{2}(\mathbb{R})$ is given by

$$
x(t)=\sum_{k} a_{J, k} \phi_{J, k}(t)+\sum_{k} d_{J, k} \psi_{J, k}(t)+\cdots+\sum_{k} d_{1, k} \psi_{1, k}(t),
$$

where $J$ is the number of multiresolution levels (or scales) and $k$ ranges from 1 to the number of coefficients in the specified level. The coefficients $a_{J, k}, d_{J, k}, \ldots, d_{1, k}$ in (7) are the wavelet transform coefficients and can be approximated by the following relations:

$$
\begin{aligned}
& a_{J, k}=\int_{-\infty}^{\infty} x(t) \phi_{J, k}(t) \mathrm{d} t, \\
& d_{j, k}=\int_{-\infty}^{\infty} x(t) \psi_{j, k}(t) \mathrm{d} t, \quad j=1,2, \ldots, J .
\end{aligned}
$$

The coefficients $a_{J, k}$, known as the smooth coefficients represent the underlying smooth behaviour of the time series at the coarse scale $2 J$, while $d_{j, k}$, known as the detailed coefficient, describes the coarse scale deviations from the smooth behaviour and $d_{J-1, k}, \ldots, d_{1, k}$ provides progressively finer scale deviations from the smooth behaviour.

The Maximal overlap discrete wavelet transform (MODWT) (Percival and Walden [21]) is similar to DWT in that high-pass and low-pass filters are applied to the input signal at each level. However, in the MODWT, the output signal is not subsampled (not decimated) but the filters are upsampled at each level. MODWT is preferred over the DWT, since the MODWT can handle any sample size $T$, whilst the DWT of level $J$ restricts the sample size to a multiple of $2^{j}$. Also MODWT (wavelet and scaling coefficients) is invariant to circularly shifting the time series under study and its multiresolution detail and smooth coefficients are associated with zero phase filters, two properties that the DWT does not hold and excepting the last few coefficients, the MODWT is not affected by the arrival of new information. Finally, the MODWT wavelet variance estimator is asymptotically more efficient than the same estimator based on DWT, which in turn makes it more suitable when calculating wavelet correlations. More details on MODWT can be found in Percival and Walden [21].

Mathematically, decomposing a time series $x(t)$ using the MODWT to $J$-levels involves the application of $J$ pairs of filters. The filtering operation at the $j$ th level consists of applying a rescaled father wavelet to yield a set of detail coefficients

$$
\widetilde{D}_{j, t}=\sum_{t=L_{j}-1}^{N-1} \widetilde{\psi}_{j, l} x_{t-l}
$$

and a rescaled mother wavelet to yield a set of scaling coefficients

$$
\widetilde{A}_{j, t}=\sum_{t=L_{j}-1}^{N-1} \widetilde{\phi}_{j, l} x_{t-l}
$$

for all times $t \in \mathbb{Z}$, where $x$ is the function to be decomposed. The rescaled mother $\widetilde{\psi}_{j, l}=\psi_{(j, l)} / 2^{j}$ and father wavelets $\widetilde{\phi}_{j, l}=$ $\phi_{j, l} / 2^{j}$ for the $j$ th level are a set of scale dependent localized differencing and averaging operators and can be regarded as rescaled versions of the originals. The $j$ th level equivalent filter coefficients have a width $L_{j}=\left(2^{j}-1\right)(L-1)+1$, where $L$ is the width of the $j=1$ base filter. 
2.2. The Wavelet Correlation and Cross Correlation. The wavelet variance $\sigma_{X}\left(\lambda_{j}\right)$ is defined as the expected value of $\widetilde{D}_{j, t}^{2}$ if we consider only the nonboundary coefficients. An unbiased estimator of the wavelet variance is formed by removing all the coefficients that are affected by the boundary conditions and is given by

$$
\sigma_{X}\left(\lambda_{j}\right)=\operatorname{Var}_{X}^{2}\left(w_{j, t}^{X}\right)=\frac{1}{M_{j}} \sum_{t=L_{j}-1}^{N-1} \widetilde{D}_{j, t}^{2}
$$

where $M_{j}=N-L_{j}+1$ is the number of nonboundary coefficients at the $j$ th level. Similarly, the wavelet covariance between functions $x(t)$ and $y(t)$ is defined to be the covariance of the wavelet coefficients at a given scale. The unbiased estimator of the wavelet covariance at the $j$ th level is given by

$$
\gamma_{X Y}\left(\lambda_{j}\right)=\operatorname{Cov}_{X Y}\left(w_{j, t}^{X}, w_{j, t}^{Y}\right)=\frac{1}{M_{j}} \sum_{t=L_{j}-1}^{N-1} \widetilde{D}_{j, t}^{2} \widetilde{D}_{j, t}^{2},
$$

where all the wavelet coefficients affected by the boundary are removed. Since the wavelet correlation estimator is usually constructed by the MODWT and is simply made up of the wavelet covariance and the wavelet variance for $X_{t}$ and $Y_{t}$, The MODWT estimator of the wavelet correlation can be expressed as

$$
\rho_{X Y}\left(\lambda_{j}\right)=\operatorname{Corr}_{X Y}\left(w_{j, t}^{X}, w_{j, t}^{Y}\right)=\frac{\operatorname{Cov}_{X Y}\left(w_{j, t}^{X}, w_{j, t}^{Y}\right)}{\sqrt{\operatorname{Var}\left(w_{j, t}^{X}\right) \operatorname{Var}\left(w_{j, t}^{Y}\right)}} .
$$

The wavelet cross-correlation estimator is a natural extension of the wavelet correlation estimator and in fact has similar properties (see Percival and Walden [21].)

\subsection{The Wavelet Multiple Correlation and Cross Correlation.} In the existing wavelet literature, the comovement of macroeconomic variables within the multivariate set of variables has been tested mostly by using pairwise correlations and cross correlations. However, owing to its several limitations the wavelet multiple correlation and cross-correlation methodology has been recently proposed by Fernández-Macho [18] to study Eurozone stock market returns.

In this novel methodology, for any multivariate stochastic process $X_{t}=\left(x_{1 t}, x_{2 t}, \ldots, x_{n t}\right)$, the wavelet coefficients $W_{j t}=\left(w_{1 j t}, w_{2 j t}, \ldots, w_{n j t}\right)$ are obtained by applying the MODWT to each $x_{i t}$ process at scale $\lambda_{j}$. The wavelet multiple correlation (WMC) $\rho_{X}\left(\lambda_{j}\right)$, for the stochastic process $X_{t}$, at each scale $\lambda_{j}$ is defined as the square root of the regression coefficient of determination in that linear combination of variables $w_{1 j t}, i=1, \ldots, n$, for which such coefficient of determination is maximum. As we know, the coefficient of determination corresponding to the regression of a variable $z_{i}$ on a set of regressors, $\left\{z_{k}, k \neq i\right\}$, is obtained by the relation $R^{2}=1-1 / \rho^{i i}$, where $\rho^{i i}$ is the $i$ th diagonal element of the inverse of the complete correlation matrix $P$.
Therefore, the equation for WMC $\rho_{X}\left(\lambda_{j}\right)$ can be formulated as

$$
\rho_{X}\left(\lambda_{j}\right)=\sqrt{1-\frac{1}{\max \operatorname{diag} P_{j}^{-1}}}
$$

where $P_{j}$ refers to the $n \times n$ correlation matrix of $W_{j t}$, and the max. $\operatorname{diag}(\cdot)$ operator provides selection for the largest element in the diagonal of the argument. Alternatively, one can also define the WMC $\rho_{X}\left(\lambda_{j}\right)$ equation in terms of wavelet variances and covariance as

$$
\rho_{X}\left(\lambda_{j}\right)=\operatorname{Corr}\left(w_{i j t}, \widehat{w}_{i j t}\right)=\frac{\operatorname{Cov}\left(w_{i j t}, \widehat{w}_{i j t}\right)}{\sqrt{\operatorname{Var}\left(w_{i j t}\right) \operatorname{Var}\left(\widehat{w}_{i j t}\right)}},
$$

where $w_{i j}$ is chosen so as to maximize $\rho_{X}\left(\lambda_{j}\right)$ and $\widehat{w}_{i j}$ are the fitted values in the regression of $w_{i j}$ on the rest of wavelet coefficients at scale $\lambda_{j}$.

Similarly, we may also define the wavelet multiple cross correlation (WMCC) as

$$
\rho_{X, \tau}\left(\lambda_{j}\right)=\operatorname{Corr}\left(w_{i j t}, \widehat{w}_{i j t+\tau}\right)=\frac{\operatorname{Cov}\left(w_{i j t}, \widehat{w}_{i j t+\tau}\right)}{\sqrt{\operatorname{Var}\left(w_{i j t}\right) \operatorname{Var}\left(\widehat{w}_{i j t+\tau}\right)}}
$$

where $\tau$ is a lag between observed and fitted values of the variable selected as the criterion variable at each scale $\lambda_{j}$. Further, it is clear from (15) and (16) that, for $n=$ 2 , the WMC and WMCC will coincide with the standard wavelet correlation and cross correlation because at the scale $\lambda_{j}, \operatorname{Cov}\left(w_{1 j t}, \widehat{w}_{1 j t}\right)=\widehat{\beta}_{j} \operatorname{Cov}\left(w_{1 j t}, w_{2 j t}\right)$ and $\operatorname{Var}\left(\widehat{w}_{1 j t}\right)=$ $\widehat{\beta}_{j}^{2} \operatorname{Var}\left(w_{2 j t}\right)$, where $\widehat{\beta}_{j}$ is the estimated coefficient in the regression of $w_{1 j t}$ on $w_{2 j t}$.

Let $X=\left\{X_{1}, X_{2}, \ldots, X_{N}\right\}$ be the realization of the multivariate stochastic process $X_{t}, t=1,2, \ldots, N$. Then, after applying the MODWT of order $J$ to each of the univariate time series $\left\{x_{i 1}, x_{i 2}, \ldots, x_{i N}\right\}, i=1,2, \ldots, n$, we would obtain $J$ length- $N$ vectors of MODWT coefficients

$$
\begin{aligned}
\widetilde{W}_{j} & =\left\{\widetilde{W}_{j 0}, \widetilde{W}_{j 0}, \ldots, \widetilde{W}_{j, N-1}\right\} \\
= & \left\{\left(\widetilde{w}_{1 j 0}, \ldots, \widetilde{w}_{n j 0}\right), \ldots,\left(\widetilde{w}_{1 j, N / 2^{j}-1}, \ldots, \widetilde{w}_{n j, N / 2^{j}-1}\right)\right\} \\
& j=1,2, \ldots, J .
\end{aligned}
$$

Therefore, based on either (14) or (15), a consistent wavelet multiple correlation estimator is given by

$$
\begin{aligned}
\tilde{\rho}_{X}\left(\lambda_{j}\right) & =\sqrt{1-\frac{1}{\max \operatorname{diag} P_{j}^{-1}}}=\operatorname{Corr}\left(\widetilde{w}_{i j t}, \widehat{\widetilde{w}}_{i j t}\right) \\
& =\frac{\operatorname{Cov}\left(\widetilde{w}_{i j t}, \widehat{\widetilde{w}}_{i j t}\right)}{\sqrt{\operatorname{Var}\left(\widetilde{w}_{i j t}\right) \operatorname{Var}\left(\widehat{\widetilde{w}}_{i j t}\right)}},
\end{aligned}
$$


TABlE 1: Descriptive statistics of Eurozone long term interest rates.

\begin{tabular}{lccccccccccc}
\hline & Germany & France & Belgium & Spain & Finland & Austria & Greece & Ireland & Portugal & Netherlands & Italy \\
\hline Mean & 3.63 & 3.91 & 4.12 & 4.44 & 3.84 & 3.96 & 7.18 & 5.08 & 5.37 & 3.82 & 4.55 \\
Median & 3.82 & 3.95 & 4.13 & 4.25 & 3.94 & 4.03 & 4.79 & 4.59 & 4.48 & 3.93 & 4.45 \\
Maximum & 5.17 & 5.26 & 5.38 & 6.79 & 5.41 & 5.37 & 29.24 & 12.45 & 13.85 & 5.3 & 7.06 \\
Minimum & 1.24 & 2.12 & 2.54 & 3.09 & 1.55 & 1.97 & 3.3 & 3.04 & 3.19 & 1.75 & 3.29 \\
Std. Dev. & 0.91 & 0.68 & 0.6 & 0.74 & 0.84 & 0.73 & 5.77 & 1.72 & 2.44 & 0.81 & 0.68 \\
Skewness & -0.7 & -0.11 & 0.06 & 0.75 & -0.49 & -0.25 & 2.24 & 1.94 & 2.03 & -0.49 & 0.88 \\
Kurtosis & 3.07 & 2.69 & 2.87 & 3.35 & 3.07 & 2.98 & 7.17 & 6.88 & 6.01 & 2.94 & 4.21 \\
Jarque-Bera & 11.68 & 0.87 & 0.19 & 13.91 & 5.89 & 1.48 & 221.1 & 178.1 & 150.6 & 5.87 & 27.11 \\
$P$ value & 0 & 0.64 & 0.9 & 0 & 0.05 & 0.47 & 0 & 0 & 0 & 0.05 & 0 \\
\hline
\end{tabular}

where the wavelet variances and covariances similar to (11), (12), and (13) are given

$$
\begin{gathered}
\operatorname{Var}\left(\widetilde{w}_{i j t}\right)=\frac{1}{\widetilde{M}_{j}} \sum_{t=L_{j}-1}^{N-1} \widetilde{w}_{i j t}^{2}, \\
\operatorname{Var}\left(\widehat{\widetilde{w}}_{i j t}\right)=\frac{1}{\widetilde{M}_{j}} \sum_{t=L_{j}-1}^{N-1} \widehat{\widehat{w}}_{i j t}^{2}, \\
\operatorname{Cov}\left(\widetilde{w}_{i j t}, \widehat{\widetilde{w}}_{i j t}\right)=\frac{1}{\widetilde{M}_{j}} \sum_{t=L_{j}-1}^{N-1} \widetilde{w}_{i j t} \widehat{\widetilde{w}}_{i j t}
\end{gathered}
$$

respectively, with $\widetilde{M}_{j}=N-L_{j}+1$. Similarly, the consistent wavelet multiple cross correlation estimator may be defined as

$$
\tilde{\rho}_{X, \tau}\left(\lambda_{j}\right)=\operatorname{Corr}\left(\widetilde{w}_{i j t}, \widehat{\widetilde{w}}_{i j t+\tau}\right)=\frac{\operatorname{Cov}\left(\widetilde{w}_{i j t}, \widehat{\widehat{w}}_{i j t+\tau}\right)}{\sqrt{\operatorname{Var}\left(\widetilde{w}_{i j t}\right) \operatorname{Var}\left(\widehat{\widetilde{w}}_{i j t+\tau}\right)}} .
$$

The confidence intervals are constructed by assuming that $X$ is a realization of $X_{t}$ with $\widetilde{W}_{j}$ as the vectors of wavelet coefficients given by (17). Also, if $\widetilde{\rho}_{X}\left(\lambda_{j}\right)$ represents the wavelet correlation obtained by (18), then

$$
\widetilde{z}_{j} \sim \mathscr{F} \mathscr{N}\left(z_{j}, N / 2^{j}-3\right)
$$

where $z_{j}=\operatorname{arctanh}\left(\rho_{X}\left(\lambda_{j}\right)\right), \widetilde{z}_{j}=\operatorname{arctanh}\left(\widetilde{\rho}_{X}\left(\lambda_{j}\right)\right)$, and $\mathscr{F} \mathcal{N}$ stands for the folded normal distribution (see FernándezMacho [18]). Therefore, the confidence intervals (CI) associated with the sample wavelet correlation coefficient can be constructed by the following relation:

$$
\mathrm{CI}_{(1-\alpha)}\left(\rho_{X}\left(\lambda_{j}\right)\right)=\tanh \left\{\tilde{z}_{j} \pm \frac{\lambda_{1-\alpha / 2}^{-1}}{\sqrt{N / 2^{j}-3}}\right\} .
$$

\section{Data Description, Results, and Discussion}

In this section we use the wavelet multiple correlation and wavelet multiple cross correlation with data from the eleven main Eurozone fixed income markets as follows: Germany, France, Italy, Spain, Netherlands, Belgium, Austria, Greece,
TABLE 2: Time interpretation of different frequencies.

\begin{tabular}{lc}
\hline$w_{i 1}$ & $2-4$ months \\
$w_{i 2}$ & $4-8$ months \\
$w_{i 3}$ & $8-16$ months \\
$w_{i 4}$ & $16-32$ months \\
\hline
\end{tabular}

Finland, Portugal, and Ireland. The monthly data of long term interest rates from January 2001 to September 2012 have been collected, considering that this period coincides with the formation of European Monetary Union. The data have been taken from the website of European central bank. Selected descriptive statistics of long term interest rates for all the stock market indices are presented in Table 1. The sample mean of long term interest rate is positive for all the eleven stock market indices under consideration. The measure of skewness indicates that the long term interest rates for Germany, France, Finland, Austria, and Netherlands are negative and for the rest of stock indices it skewed positively. The distribution of long term interest rate exhibits excess kurtosis for stock indices of Germany, Spain, Finland, Greece, Ireland, Portugal, and Italy, which indicate that data series are leptokurtic relative to normal distribution. However, for rest of the stock indices the distributions are platykurtic in character. Excluding France, Belgium, and Austria, the Jarque-Bera normality test was rejected for all the countries.

In order to calculate the wavelet multiple correlation we begin by decomposing the long-term bond rates into different time scales using MODWT to each of the long term interest rate series. Considering the smaller sample size, we decomposed the monthly long-term interest rates by applying the MODWT with a Daubechies least asymmetric (LA) wavelet filter of length $L=4$, commonly denoted by $L A$ (4). This filter is favored because of our small sample size.

Given the maximum decomposition level $\log _{2}(T)$, we could have attained the maximum level of seven decompositions. However, the number of feasible wavelet coefficients gets small for higher levels; we therefore chose to carry out the wavelet analysis with $J=4$ so that four wavelet coefficients and one scaling coefficient were produced for each country, that is, $w_{1 i}, w_{2 i}, w_{3 i}, w_{4 i}$, and $v_{4 i}$, respectively. The time dynamics of each wavelet coefficient in Table 2 is given by $\left[2^{j},-2^{j+1}\right]$ (Since frequency and time are inversely related, 
TABLE 3: Wavelet multiple correlation and multiple cross correlation within $95 \%$ confidence intervals.

\begin{tabular}{|c|c|c|c|c|c|c|c|c|c|c|c|c|c|c|c|}
\hline Scales $\downarrow$ & Lower & Cor & Upper & Lower & Cor & Upper & Lower & Cor & Upper & Lower & Cor & Upper & Lower & Cor & Upper \\
\hline Lags $\rightarrow$ & & -10 & & & -5 & & & 0 & & & +5 & & & +10 & \\
\hline$w_{i 1}$ & 0.16 & 0.38 & 0.57 & 0.22 & 0.43 & 0.60 & 0.96 & 0.97 & 0.98 & -0.03 & 0.19 & 0.41 & -0.01 & 0.21 & 0.42 \\
\hline$w_{i 2}$ & 0.11 & 0.43 & 0.66 & 0.35 & 0.61 & 0.78 & 0.97 & 0.98 & 0.99 & 0.18 & 0.48 & 0.70 & 0.00 & 0.33 & 0.60 \\
\hline$w_{i 3}$ & 0.06 & 0.53 & 0.80 & 0.40 & 0.73 & 0.89 & 0.99 & 0.99 & 0.99 & 0.35 & 0.71 & 0.88 & 0.05 & 0.52 & 0.80 \\
\hline$w_{i 4}$ & 0.61 & 0.92 & 0.98 & 0.53 & 0.89 & 0.98 & 0.99 & 0.99 & 0.99 & 0.34 & 0.84 & 0.97 & -0.03 & 0.68 & 0.93 \\
\hline Lags $\rightarrow$ & & -20 & & & -15 & & & 0 & & & +15 & & & +20 & \\
\hline$w_{i 1}$ & -0.00 & 0.23 & 0.44 & 0.18 & 0.40 & 0.58 & 0.96 & 0.97 & 0.98 & 0.17 & 0.39 & 0.51 & 0.09 & 0.32 & 0.51 \\
\hline$w_{i 2}$ & 0.18 & 0.48 & 0.70 & 0.15 & 0.46 & 0.68 & 0.97 & 0.98 & 0.99 & 0.26 & 0.55 & 0.67 & 0.12 & 0.44 & 0.67 \\
\hline$w_{i 3}$ & 0.31 & 0.68 & 0.87 & 0.15 & 0.59 & 0.83 & 0.99 & 0.99 & 0.99 & 0.41 & 0.74 & 0.92 & 0.52 & 0.80 & 0.92 \\
\hline$w_{i 4}$ & 0.54 & 0.90 & 0.98 & 0.32 & 0.83 & 0.96 & 0.99 & 0.99 & 0.99 & 0.13 & 0.76 & 0.96 & 0.32 & 0.83 & 0.96 \\
\hline
\end{tabular}

Cor $=$ correlation coefficient, Lower $=$ lower bound of $95 \%$ confidence Interval, Upper $=$ upper bound of $95 \%$ confidence interval.

the $J$ wavelet coefficients are associated with the frequency interval given by $\left[1 / 2^{j}, 1 / 2^{j+1}\right]$.). The first wavelet coefficient therefore represents the dynamics of time series within two to four months, the second one within four to eight months, and so on. Wavelet multiple correlation within 95\% confidence intervals obtained from all the bond rates is shown in Figure 1 and Table 3. It is clear from the correlation plots and the ninth column of Table 3 that multiple correlations are significant and high at all the timescales (Results should, however, be interpreted with caution. Confidence intervals are based on fisher's result for usual bivariate correlation.). We find that correlation is strong at all the frequencies and this correlation grows stronger with lower frequencies. In particular correlation at the highest frequency (16 32 months) is nearly perfect. The discrepancies between Eurozone bond markets are therefore small and negligible. The wavelet multiple cross correlations for the different wavelet scales with leads and lags are presented in Figure 2 and Table 3. The country that maximizes the multiple correlation against the linear combination of other countries is shown in the upper right corner of Figure 2. We find that multiple cross correlations get stronger with lower frequencies and France maximizes the multiple correlations against the linear combination of other countries. This indicates that France has a potential to lead or lag other markets at all the studied frequencies. However, given the symmetry (zero skewness) in Figure 2, it is difficult to say whether French fixed income market leads or lags the linear combination of other markets. Overall, it is found that Eurozone fixed income markets are nearly perfectly integrated especially at higher time scales.

The investigation of fixed income market linkages in international financial markets is of utmost importance. It has important policy implications for monetary policy independence, modeling long term interest rates, bond portfolio diversification, and fiscal deficit financing. While the conventional correlation has often been used to study the market integration for different countries, it does not reveal the correlation at different time horizons. We study fixed income market integration at different time horizons by exploiting the methodology of wavelets. Our results show that the fixed income markets in Eurozone are highly integrated

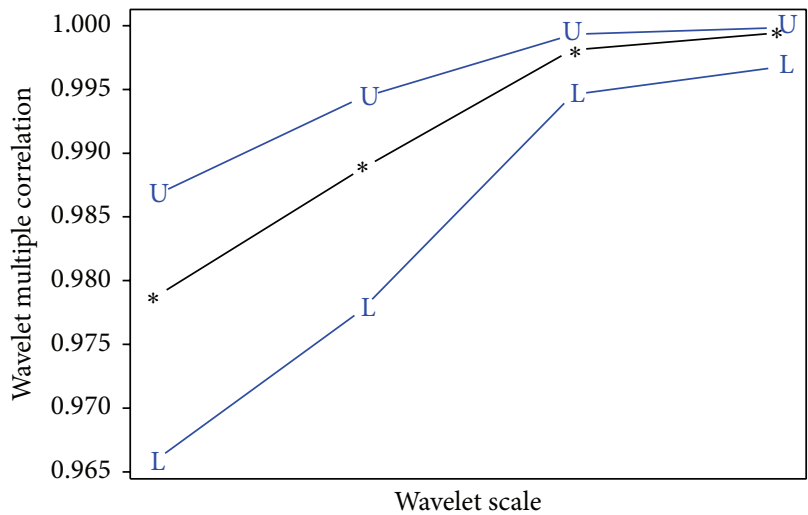

FIgURE 1: Wavelet multiple correlations for the Eurozone bond market at different time scales. The coloured lines correspond to the upper and lower bounds of the $95 \%$ confidence interval.

at all the studied frequencies. The Eurozone fixed markets behave as a single coherent market. The synchronization in these countries therefore provides very little scope of independent monetary policy by member countries. Monetary policy, in essence, enables the central bank to react to the specific problems of the economy. For example, to curb the internal and external demand and supply shocks and subsequently create an ambience of price stability and overall economic stability, monetary policy is important. However, the formation of Eurozone contributes a significant loss to the independence of monetary policy making by member countries. Further, given the very high correlation at different frequencies, there is almost no scope for bond diversification in Eurozone countries. This study could be extended to analyze the dynamic comovement of bond markets during the tranquil as well as recession periods.

\section{Conclusion and Policy Implications}

We applied a new methodology based on wavelet multiple correlation and wavelet multiple cross correlation to study the integration in the Eurozone fixed income markets. Our 



Figure 2: Wavelet multiple cross-correlations for the Eurozone Bond markets at different time scales (with France acting as potential leader/follower). The coloured lines correspond to the upper and lower bounds of the $95 \%$ confidence interval.

results based on wavelet multiple correlation and cross correlation indicated that Eurozone fixed income markets are highly integrated with integration becoming stronger with lower frequencies. At the lowest frequency (long run) corresponding to time dynamics of 16 32 months, Eurozone fixed income markets were found to be almost perfectly integrated. These results are the unique contribution of this study which we have been able to calculate with single shot. Further, we allowed certain number of lags between observed and fitted values from the same linear combination as before at each of the wavelet scales. Our results in particular showed that French fixed income market maximizes the multiple correlation against the linear combination of other countries, hence indicating that France has a potential to lead or lag the linear combination of other markets. Over all, we found strong to very strong linkages in the Eurozone fixed income markets, especially at lower frequencies. From the perspective of national central banks in Eurozone area, we conclude that there is less scope for independent monetary policy. Also, a very high correlation at different frequencies indicates that there is almost no scope for bond diversification in Eurozone countries.

\section{Conflict of Interests}

The authors declare that there is no conflict of interests regarding the publication of this paper. 


\section{References}

[1] P. Hartmann, A. Maddaloni, and S. Manganelli, "The euro-area financial system: structure, integration, and policy initiatives," Oxford Review of Economic Policy, vol. 19, no. 1, pp. 180-213, 2003.

[2] S. M. Bartram and Y. Wang, "Another look at the relationship between cross-market correlation and volatility," Finance Research Letters, vol. 2, no. 2, pp. 75-88, 2005.

[3] G. Hardouvelis, D. Malliaropulos, and R. Priestley, "EMU and European stock market integration," Journal of Business, vol. 79, no. 1, pp. 365-391, 2006.

[4] P. F. Diamandis, "International stock market linkages: evidence from Latin America," Global Finance Journal, vol. 20, no. 1, pp. 13-30, 2009.

[5] D. M. Hunter and D. P. Simson, "A conditional assessment of the relationships between the major world bond markets," European Financial Management, vol. 11, pp. 463-482, 2005.

[6] M. R. Barassi, G. M. Caporale, and S. G. Hall, "Irreducibility and structural cointegrating relations: an application to the G7 long-term interest rates," International Journal of Finance and Economics, vol. 6, no. 2, pp. 127-138, 2001.

[7] R. A. Fujihara and M. Mougoué, "International linkages between short-term real interest rates," Quarterly Review of Economics and Finance, vol. 36, no. 4, pp. 451-473, 1996.

[8] R. P. Degennaro, R. A. Kunkel, and J. Lee, "Modeling international long-term interest rates," Financial Review, vol. 29, no. 4, pp. 577-597, 1994.

[9] B. Kiran, "Integration of long-term interest rates: empirical evidence for G7 countries," Global Economic Review, vol. 41, no. 3, pp. 279-290, 2012.

[10] K. Smith, "Government bond market seasonality, diversification, and cointegration international evidence," The Journal of Financial Research, vol. 25, no. 2, pp. 203-221, 2002.

[11] J. Yang, "International bond market linkages: a structural VAR analysis," Journal of International Financial Markets, Institutions and Money, vol. 15, no. 1, pp. 39-54, 2005.

[12] A. B. Dar, N. Bhanja, A. Samantaraya, and A. K. Tiwari, "Export led growth or growth led export hypothesis in Indiaevidence based on time-frequency approach," Asian Economic and Financial Review, vol. 3, no. 7, pp. 869-880, 2013.

[13] A. B. Dar, A. Samantaraya, and F. A. Shah, "The predictive power of yield spread: evidence from wavelet analysis," Empirical Economics, vol. 46, no. 3, pp. 887-901, 2014.

[14] A. K. Tiwari, A. B. Dar, N. Bhanja, and A. Shah, "Stock market integration in Asian countries: evidence from wavelet multiple correlations," Journal of Economic Integration, vol. 28, no. 3, pp. 441-456, 2013.

[15] A. Rua and L. C. Nunes, "International comovement of stock market returns: a wavelet analysis," Journal of Empirical Finance, vol. 16, no. 4, pp. 632-639, 2009.

[16] M. Raghavan, J. Dark, and E. A. Maharaj, "Impact of capital control measures on the Malaysian stock market: a multiresolution analysis," International Journal of Managerial Finance, vol. 6, no. 2, pp. 116-127, 2010.

[17] J. Cohen, P. Cohen, S. West, and L. Aitken, Applied Multiple Regression and Correlation Analysis, vol. 1, Lawrence Erlbaum Associates, New Jersey, NJ, USA, 3rd edition, 2003.

[18] J. Fernández-Macho, "Wavelet multiple correlation and crosscorrelation: a multiscale analysis of Eurozone stock markets," Physica A: Statistical Mechanics and its Applications, vol. 391, no. 4, pp. 1097-1104, 2012.
[19] L. Aguiar-Conraria, N. Azevedo, and M. J. Soares, "Using wavelets to decompose the time-frequency effects of monetary policy," Physica A: Statistical Mechanics and Its Applications, vol. 387, no. 12, pp. 2863-2878, 2008.

[20] I. Daubechies, Ten Lectures on Wavelets, SIAM, Philadelphia, Pa, USA, 1992.

[21] D. B. Percival and A. T. Walden, Wavelet Methods for Time Series Analysis, Cambridge University Press, Cambridge, UK, 2000. 

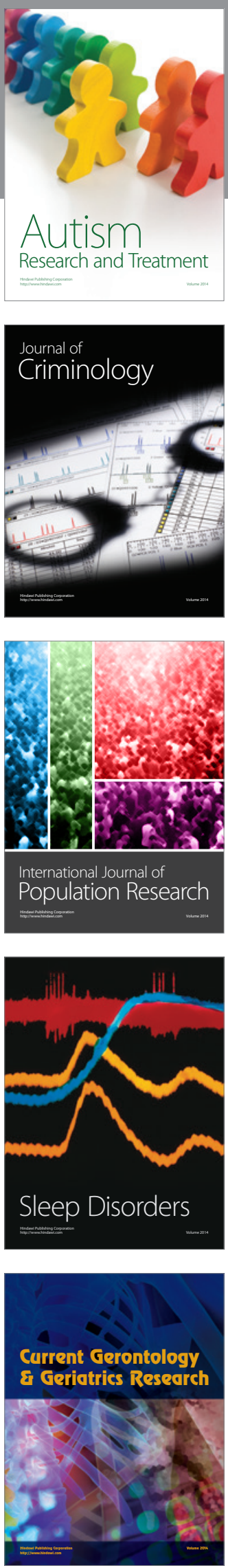
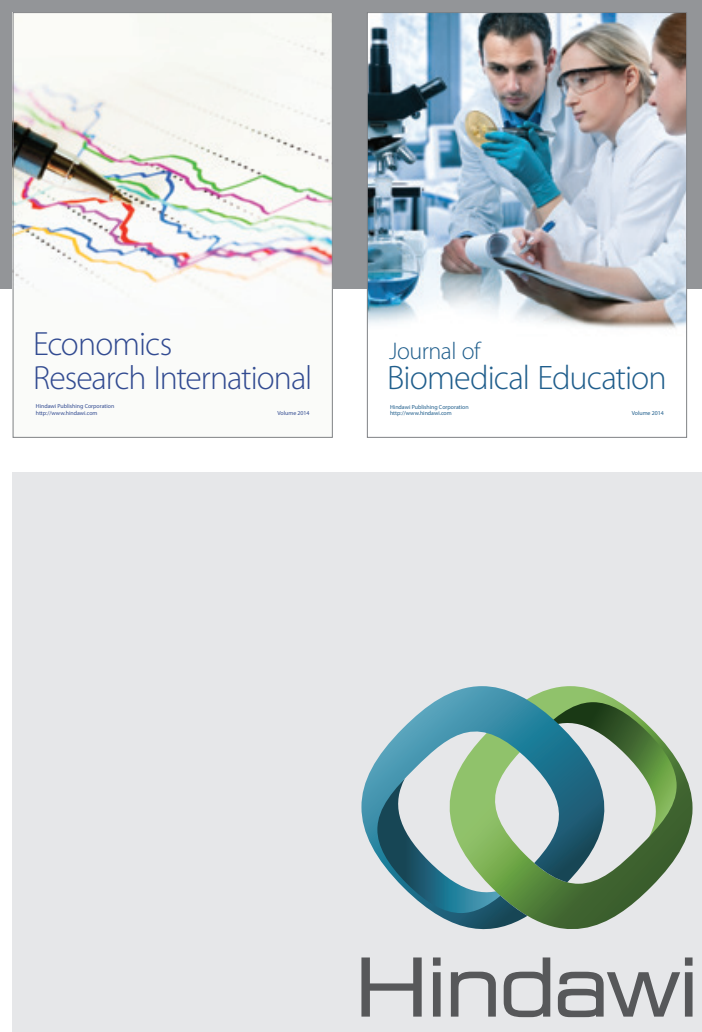

Submit your manuscripts at

http://www.hindawi.com
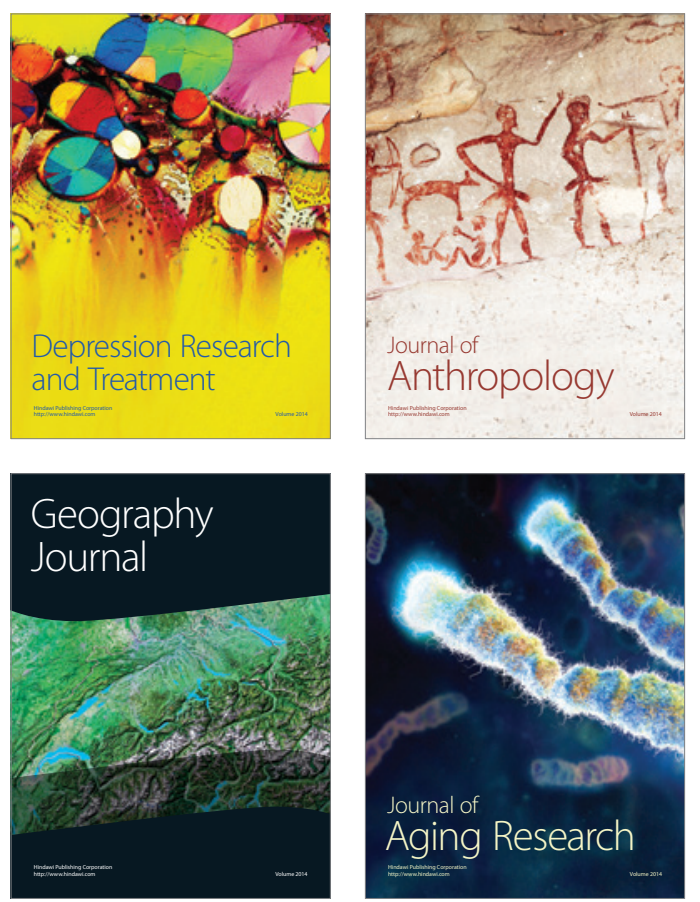
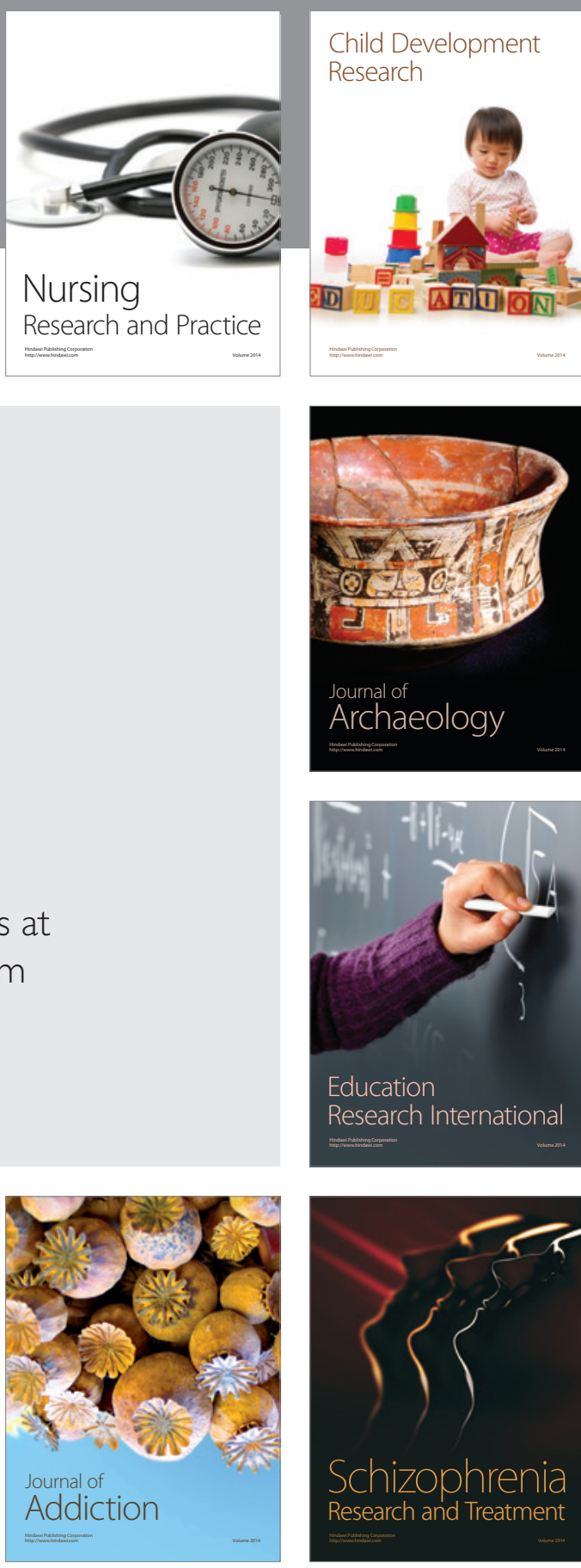

(D)
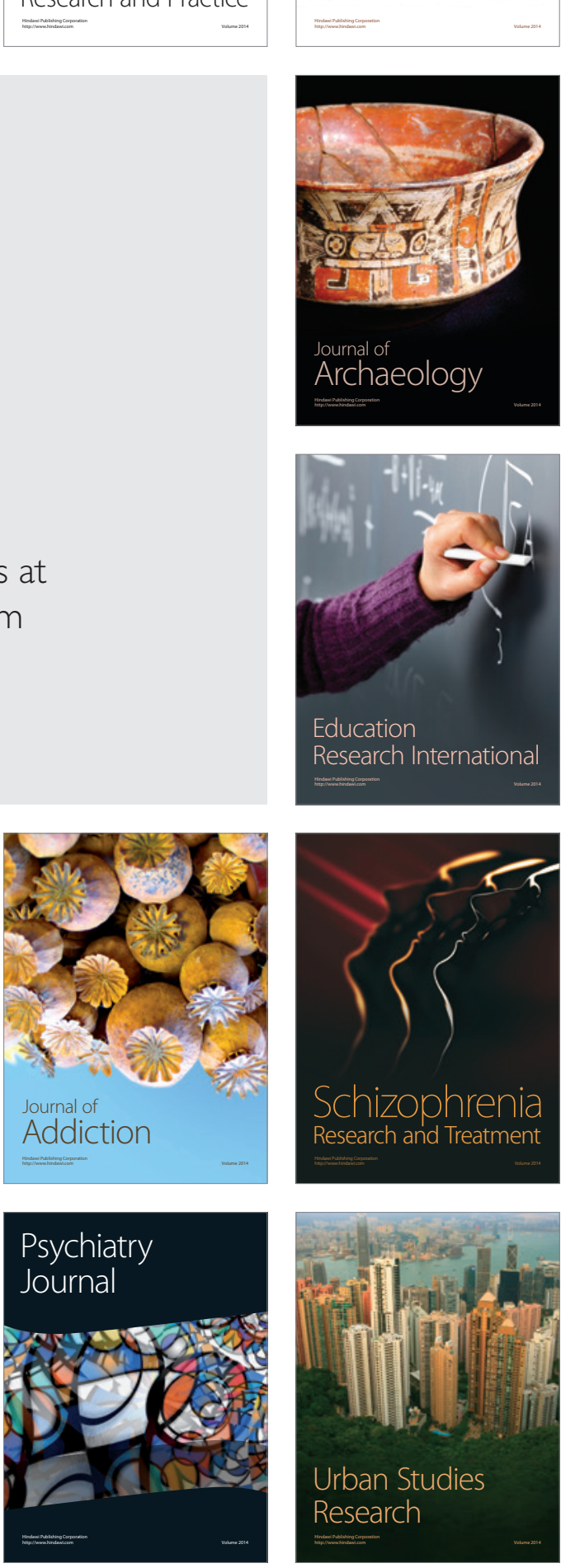